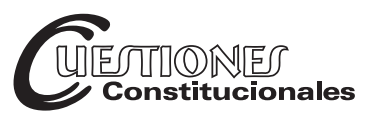

Revista Mexicana de Derecho Constitucional

Núm. 41, julio-diciembre 2019

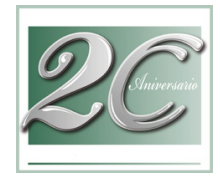

\title{
Fundamentación ética del derecho a la soberanía alimentaria en Colombia
}

\author{
Ethical justification of the law to food sovereignty \\ in Colombia
}

\author{
María Alejandra RAMÍREZ* \\ Libia Esperanza NIETO GÓMEZ** \\ Reinaldo GIRALDO DÍAZ***
}

\begin{abstract}
Y si somos Severinos iguales en todo en la vida, morimos de muerte igual, la misma muerte Severina: que es la muerte de quien se muere de viejo antes de los treinta, de emboscada antes de los veinte de hambre un poco cada día de la debilidad y la enfermedad, es que la muerte Severina ataca a cualquier edad hasta a la gente no nacida.
\end{abstract}

\section{João CABRAL}

\footnotetext{
* Abogada. Investigadora en la Universidad Libre Seccional Cali, Colombia. Grupo de Investigación "Ignacio Torres Giraldo"; maralejramirez@gmail.com.

** Ingeniera agrícola, especialista en recursos hidráulicos por la Universidad Nacional de Colombia, Colombia. Docente investigadora en la Universidad Nacional Abierta y a Distancia (UNAD), Bogotá, Colombia; libia.nieto@unad.edu.co.

*** Doctor en filosofía, Universidad de Antioquia, Colombia. Ingeniero agrónomo por la Universidad Nacional de Colombia, Colombia. Docente asistente en la Universidad Nacional Abierta y a Distancia, Colombia; reinaldo.giraldo@unad.edu.co.
} 
Esta revista forma parte del acervo de la Biblioteca Jurídica Virtual del Instituto de Investigaciones Jurídicas de la UNAM

RESUMEN: El presente artículo hace algunas precisiones sobre el contexto actual frente al que se mueven los derechos humanos, centrándose específicamente en el caso colombiano y el estado de reconocimiento a la soberanía alimentaria de las comunidades y algunas apuestas desde los territorios que resisten, dentro de un modelo que promueve la globalización económica y que no tiene en cuenta las necesidades locales y comunitarias. Desde la ética, como posibilidad de fundamentación y las nuevas conquistas políticas de los pueblos de América Latina en las últimas décadas, producto de la resistencia en contra del menoscabo de la vida y en pro de la defensa del territorio, es pertinente reflexionar sobre los derechos humanos y realizar un análisis que permita entender la necesidad de reformularlos, de acuerdo con el contexto actual en las comunidades que le apuestan a una lucha por la supervivencia, en tal sentido y para el caso en concreto, se fundamentará en torno al derecho de los pueblos a la soberanía alimentaria.

Palabras clave: globalización, organismos multilaterales, derechos humanos, soberanía alimentaria.
ABSTRACT: The present article does some precisions on the current context opposite to which the human rights move, centering specially on the Colombian case and the state of recognition to the food sovereignty of the communities and some bets from the territories that resist, inside a model who promotes the economic globalization and who does not bear in mind the local and community needs. From the ethics as possibility of justification and the new political conquests of the peoples of America Latina in the last decades, product of the resistance against the impairment of the life and the advantage of the defense of the territory, is pertinent to reflect on the human rights and to realize an analysis which will allow understand the need of reformulate it, in accordance on the current context of the communities which have bet on a struggle for the survival, in this sense and for the case in particular, it will be based concerning the right of the peoples to the food sovereignty.

Keywords: globalization, multilateral agencies, human rights, food sovereignty

SUMARIO: I. Introducción. II. Contextualización. III. Fundamentación desde la ética. IV. Seguridad y soberanía alimentaria. V. Resistencia y re-existencia desde el territorio. VI. Conclusiones. VII. Bibliografia.

\section{INTRODUCCIÓN}

Sin desconocer el peso histórico, político y social que tienen los actuales derechos humanos como conquistas políticas que en un momento de la historia fueron de utilidad para contrarrestar el poder estatal y que en la actualidad contribuyen a la defensa frente a situaciones que pretenden atentar o atentan contra la vida, es necesario realizar una crítica a los mismos, partiendo del hecho de que su razón de ser es salvaguardar al individuo y no al sujeto colectivo, lo que hace que se articule con todo un andamiaje supranacional que defiende la subjetividad visto desde un contexto 
biopolítico, ${ }^{1}$ donde el mercado es el encargado de poner en marcha la relación del sujeto tanto en el interior como en el exterior, generalizándolo, abstrayéndolo y universalizándolo.

El presente artículo hace algunas precisiones sobre el contexto actual frente al cual se mueven los derechos humanos, centrándose específicamente en el caso colombiano y el estado de reconocimiento a la soberanía alimentaria de las comunidades y algunas apuestas desde los territorios que resisten dentro de un modelo que promueve la globalización económica y que no tiene en cuenta las necesidades locales y comunitarias, todo esto orquestado desde los organismos multilaterales.

La urgencia de replantear otros derechos surge de la imperiosa necesidad de establecer medidas que permitan contrarrestar la crisis frente a la que transitan algunos pueblos de América Latina, con el fin de que se constituyan como posibles alternativas al proyecto homogeneizador de los organismos multilaterales y a la desigualdad económica que se evidencia en el alza de los precios de los alimentos y en la pérdida de la capacidad nacional de producirlos, consecuencia de la implementación de políticas neoliberales y de la proliferación de tratados de libre comercio.

En el mismo sentido, la prevalencia de la disyuntiva oferta-demanda indiscutiblemente soporta un enfoque completamente economicista de lo que significan los derechos humanos y las dificultades para garantizarlos. Derechos que se supone son fundamentales para el ser humano y su desarrollo, terminan siendo mercantilizados. Los ejemplos más claros son los de educación y salud, cuyo enfoque actual es hacia la privatización, razón por la cual se dificulta su realización; en tal sentido, la cuestión no es tan simple cuando se refiere a que el problema de los derechos humanos ya no es su justificación, sino su realización. ${ }^{2}$ Basta con mirar las vulneraciones a derechos humanos cuando desde la misma normatividad se impide el acceso y se posibilita un quiebre sustancial en la aplicación; todo esto ocurre en razón de una debilidad en la fundamentación que permite acomodar los derechos según la capacidad del Estado para materializarlos.

Por tal motivo, partiendo de las dificultades que hoy presentan los derechos humanos, es necesario preguntarse: ¿deben ser reivindicados? ¿Por qué fundamentar el derecho de los pueblos a la soberanía alimentaria? ¿Para qué elevar a derecho fundamental la soberanía alimentaria?

\footnotetext{
1 Hardt, Michael y Negri, Antonio, Imperio, Bogotá, Desde Abajo, 2000, p. 17.

2 Bobbio, Norberto, El tiempo de los derechos, Madrid, Sistema, 1991.
} 
Para responder a los cuestionamientos presentados, se planteará, desde la ética, la posibilidad de fundamentación, donde se expondrá como exigencia moral el derecho de los pueblos a la soberanía alimentaria para que se garantice una agricultura sostenible, y donde el cuidado de la madre tierra se instituya como elemento de primer orden para garantizar la supervivencia y establecer que existen bienes que no deben ser objeto del mercado, sino que, por el contrario, deben ser protegidos y garantizados por el Estado.

En Colombia, y en el resto de los países de América Latina, se vienen gestando resistencias contra lo que el mercado denomina desarrollo o progreso, que ya ha generado impactos socio-ambientales, tales como colapsos en la función hidrológica, pérdida de la biodiversidad, degradación forestal, industrialización forzosa de los pueblos, destrucción de los modos de vida, ruptura del tejido social y marginalización económica y social de las personas que se ven obligadas a desplazarse de los sectores rurales hacia los sectores urbanos. ${ }^{3}$

Menguar la crisis alimentaria que se ha ido agudizando implica atender las exigencias de las comunidades en materia de su derecho a la alimentación y trascender del concepto de seguridad alimentaria a soberanía alimentaria para que desde la normatividad se generen maneras de proteger las apuestas de vida locales y autonómicas, que se vienen gestando con el fin de proteger el territorio y la vida.

\section{CONTEXTUALIZACIÓN}

Antes de precisar sobre una fundamentación ética, se debe establecer la relación entre expansión del proyecto neoliberal y la crisis civilizatoria actual, como consecuencia del agotamiento de un modelo económico adoptado por la mayoría de países en América Latina, frente al cual es urgente tomar algunas medidas que mengüen la situación por la cual atraviesan.

La política y la economía neoliberal de los años ochenta y noventa insertaron al mundo en una crisis que es multidimensional, porque no se asiste sólo a la crisis financiera y económica, sino a una que, también, es

3 Al respecto, se sugiere consultar los trabajos de Nieto Gómez, Libia Esperanza et al., (In) Certezas del desarrollo: fisuras, relatos y otros senderos, Bogotá, Universidad Nacional Abierta y a Distancia (UNAD), 2014, disponible en: http://hemeroteca.unad. edu.co/index.php/book/article/view/1085 (fecha de acceso: 31 de enero de 2018). 
climática, energética, alimentaria y ambiental, a la que algunos autores han denominado crisis civilizatoria.

Una de las manifestaciones de la crisis civilizatoria son las expresiones de descontento manifestadas en demandas alimentarias de una gran parte de la población; en el mismo sentido, uno de los principales responsables es la agroindustria que genera altos costos ambientales y sociales, manifestados en la acumulación de tierras, desplazamiento de campesinos y comunidades ancestrales, imposición del uso de biotecnologías, monocultivos y control de la comercialización. ${ }^{4}$

Con el fin de disminuir los efectos de la crisis civilizatoria, se ha establecido una agenda bioeconómica, para impartir un nuevo orden que frene los efectos que se vienen generando, producto de la crisis; sin embargo, "La bioeconomía es un proyecto y al mismo tiempo una visión situada dentro de la narrativa neoliberal del crecimiento económico y de la competitividad y habla de una nueva economía basada en la manipulación, explotación y apropiación tecnológica de la materia viviente". ${ }^{5}$

La nueva agenda bioeconómica de la OCDE (2009) trae consigo nuevos conflictos en los territorios, debido a los impactos socio-ambientales que se ven reflejados en el deterioro de la vida, pues la apuesta es hacia la competitividad y a la productividad; lo que busca esta agenda es incentivar el desarrollo de la industria, la salud y la agricultura con base en la manipulación genética de los organismos vivos para así obtener un mayor aprovechamiento de los recursos naturales que a la larga implican destrucción, menoscabo y agotamiento de los mismos, además de la implementación de megaproyectos en materia de infraestructura y extractivos que ya han dejado víctimas del desarrollo.

El auge de la aplicación de biotecnologías obedece a la expansión del discurso neoliberal que no sólo está inmerso en un esquema económico, sino que está incrustado hasta los tuétanos en todas las legislaciones latinoamericanas, y por ende es defendido por todo el aparato estatal, por eso siempre está a la defensa del mercado sobre la vida; en ese mismo sentido,

4 Sobre este aspecto se sugiere consultar Vega Cantor, Renán, "Crisis civilizatoria", Revista Herramienta, Argentina, núm. 42, octubre de 2009, disponible en: https://www. herramienta.com.ar/articulo.php?id=1052 (fecha de acceso: 23 de marzo de 2018).

5 Pavone, Vincenzo, "Ciencia, neoliberalismo y bioeconomía", Revista Iberoamericana de Ciencia, Tecnología y Sociedad - CTS, Argentina, núm. 20, abril de 2012, pp. 1-15, disponible en: http://www.redalyc.org/articulo.oa? $i d=92424169013$ (fecha de acceso: 16 de enero de 2017) 
El neoliberalismo es más que una doctrina económica, política o social. Es economía porque estudia la regulación a través de una analítica concreta, aquella de la economía clásica. Es política, porque fundamenta al Estado moderno, la democracia, y el sistema político moderno, desde el liberalismo clásico. Es ética, porque establece los fundamentos de la convivencia social desde una posición determinada por la razón de mercado y el individualismo. Es histórica porque construye a la razón de mercado como heurística y hermenéutica de la historia. Es jurídica porque establece un modelo de Estado y de contrato social desde el cual se regula y administran las sociedades. Es simbólica porque ha generado una ideología de éxito individual sustentado en el consumo y el mercado. ${ }^{6}$

Ante la crisis que es como un precipicio frente al que se puede decidir saltar o retroceder, tomar conciencia colectiva de la injusticia debe ser una premisa $^{7}$ para que se puedan reunir las fuerzas suficientes para el cambio social; partiendo de ese punto, si bien reconsiderar el modelo económico no es una posibilidad debido a todas las presiones internacionales que esto conllevaría y a la inflexibilidad del establecimiento, se puede apostar por procesos desde lo local para hacerle frente a este modelo; de lo contrario se irá inevitablemente hacia "los límites de la Tierra son los límites terminales de este sistema que ha imperado durante varios siglos", ${ }^{8}$ y así, será la tierra la que cobre factura por la devastación que genera el pretender seguir acumulando capital.

El modelo neoliberal, llevado a la agricultura y maquinado desde la agroindustria, concentra la riqueza, genera pobreza; basta con ver el coeficiente Gini que mide los índices de desigualdad (siendo 1 el más alto); en Colombia, el Gini rural en materia de concentración de tierras se encuentra en $0,862 .{ }^{9}$

6 Dávalos, Pablo, "Neoliberalismo y Estado social de derecho", América Latina en Movimiento ALAI, Ecuador, junio de 2008, disponible en: http://www.puce.edu.ec/do cumentos/NeoliberalismoyEstadosocialdederecho.pdf (fecha de acceso: 21 de febrero de 2017).

7 Así lo señala Monedero, Juan Carlos, El gobierno de las palabras. Politica para tiempos de confusión, México, Fondo de Cultura Económica, 2011.

8 Boff, Leonardo, "El camino más corto hacia el fracaso", Rebelión, España, disponible en: http://www.rebelion.org/noticia.php? id $=84428$ (fecha de acceso: 2 de enero de 2018).

9 Instituto Geográfico Agustín Codazzi (IGAC), Atlas de la distribución de la propiedad rural en Colombia, Bogotá, Imprenta Nacional de Colombia, 2012. 
Según el Informe Nacional de Desarrollo Humano (INDH), ${ }^{10}$ se pone en la palestra al modelo agrario porque genera desigualdad en un país como Colombia, donde el $32 \%$ de los pobladores son campesinos; dicho modelo es inequitativo, hace más vulnerable a la población rural, discrimina a la mujer, excluye, no contribuye a la conservación del medio ambiente, es poco democrático, concentra la riqueza y promueve conflictos sociales y ambientales.

Colombia es un país considerablemente desigual, donde el 27,8\% de la población urbana está en situación de pobreza y el $40 \%$ de la población rural se encuentra en la misma situación, ${ }^{11}$ mientras que el 77\% de la tierra la tienen concentrada el 13\% de propietarios, con 16 millones de hectáreas de tierras aptas para la producción, sin explotar. ${ }^{12}$

Una de las propuestas ante el desolador contexto planteado es la lucha por establecer la soberanía alimentaria como un derecho de los pueblos, para que desde el mismo establecimiento sea respetado y garantizado, para que no ocurran episodios como los del municipio de Campoalegre, Huila, Colombia, donde se destruyeron más de $70 \mathrm{t}$ de arroz porque los campesinos decidieron no cultivar con semillas certificadas, como lo indica la resolución 970 de 2010 del Instituto Colombiano Agropecuario (ICA), ${ }^{13}$ o el caso de los arroceros en el departamento de Casanare, Colombia, que cultivaron con semillas contaminadas por una bacteria que destruyó todos sus cultivos y que había sido certificada por el ICA.

10 Informe Nacional de Desarrollo Humano (INDH), Colombia rural: razones para la esperanza, Programa de las Naciones Unidas para el Desarrollo, 2011.

11 Departamento Nacional de Estadística (DANE), Colombia, Encuesta sobre calidad de vida, 2015.

12 Sobre el tema de la desigualdad en Colombia, se recomienda consultar "Así es la Colombia rural", Revista Semana, Colombia, 2012, disponible en: http://especiales. semana.com/especiales/pilares-tierra/asi-es-la-colombia-rural.html (fecha de acceso: 5 de marzo de 2018); los estudios de Robinson, James, "La miseria en Colombia", Revista Desarrollo y Sociedad, Colombia, núm. 76, enero-junio de 2016, pp. 9-88, disponible en: https://revistas.uniandes.edu.co/doi/pdf/10.13043/dys.76.1 (fecha de acceso: 20 de enero de 2017); así como los desarrollos de Cárdenas, Johana y Vallejo, Luis, “Agricultura y desarrollo rural en Colombia 2011-2013: una aproximación", Apuntes del Cenes, núm. 62, diciembre de 2016, pp. 87-123, disponible en: http://www.scielo.org.co/scielo. php? script $=$ sci_arttext\&pid $=S 01200532016000200004 \& \operatorname{lng}=e n \& \operatorname{lng}$ (fecha de acceso: 27 de noviembre de 2017).

13 El Instituto Colombiano Agropecuario (ICA) es la autoridad sanitaria y de inocuidad agroalimentaria en Colombia. 


\section{FUNDAMENTACIÓN DESDE LA ÉTICA}

Fundamentar un derecho desde la ética implica considerar la vida como estética de la existencia. ${ }^{14}$ Desde esta perspectiva, la ética tiene que ver con una manera de ser, con una auto-elaboración cuyo objetivo es resistir a los mecanismos de sujeción impuestos durante siglos por Occidente. ${ }^{15}$ De allí que fundamentar éticamente el derecho a la soberanía alimentaria tiene que ver con el cómo de la vida. Un cómo que desde el capitalismo tardío está marcado por el consumo desmedido, por la crisis de valores. Un cómo que desde la fundamentación ética que se propone del derecho a la soberanía alimentaria tiene que ver con la autodeterminación de los pueblos; un cómo de la vida que resiste a la homogeneización que se impone desde Occidente, y que ocurre en todos los órdenes de la vida, incluida la imposición a sangre y fuego, y formas más sutiles, como la educación o la ideología.

Siguiendo el hilo conductor de la ética como fundamento del derecho a la soberanía alimentaria, es preciso referirse a la evolución y el ejercicio del derecho en el mundo contemporáneo, que apunta hacia la vida como objeto del poder. ${ }^{16} \mathrm{El}$ poder logra un comando efectivo sobre toda la vida de la población sólo cuando se torna una función integral, vital, que cada individuo incorpora y reactiva con su acuerdo. De ahí la necesidad de pensar no sólo en alternativas de derecho, sino alternativas al derecho. Con la propuesta de fundamentar el derecho a la soberanía alimentaria se presenta esta discusión, el poder busca producir la vida misma; y lo que se puede anteponer a este poder que infiltra la vida es la ética. De allí la importancia de fundamentar el derecho a la soberanía alimentaria desde la ética, es decir, desde la vida como obra de arte, como forma de morar en el mundo, de cuidar el mundo, de habitarlo.

14 Sánchez, Wilson et al., Ontología del presente y construcción de un carácter en Michel Foucault. Discusión a partir del pensamiento de Foucault de los problemas del presente, Santiago de Cali, Universidad Libre-Mariposas Amarillas, 2015.

15 Particularmente importantes son los aportes de Ángel, Julialba (comp.), Saber, poder y nuevas formas de lucha en Foucault, Bogotá, Universidad Nacional Abierta y a Distancia, 2016, disponible en http://hemeroteca.unad.edu.co/index.php/book/article/ view/1695BIRCH (fecha de acceso: 15 de abril de 2017), y los de Giraldo, Reinaldo, "La ética en Michel Foucault o de la posibilidad de la resistencia", Tabula Rasa, Colombia, núm. 10, enero-junio de 2009, pp. 225-241, disponible en: http://www.revistatabularasa. org/numero-10/07giraldo.pdf (fecha de acceso: 13 de marzo de 2018).

16 Hardt, Michael y Negri, Antonio, op. cit. 
La ética da cuenta del hacer de las personas en el mundo de la vida. ${ }^{17} \mathrm{La}$ definición originaria de los derechos humanos hace referencia a la comprensión de la dignidad que, inmanente a la condición humana, resulta ser anterior a juicio alguno, ya sea jurídico o político. Es un acto egregio de la persona, que hace visible las cualidades morales. Es la dignidad del ser humano que se manifiesta entre dos características básicas: libertad e igualdad plena de toda persona. ${ }^{18}$ Es necesario restituir la ética, para que más allá de los derechos civiles y políticos, sea el fundamento de lo económico, lo social y lo cultural para realizar una relectura crítica de los derechos del ser humano; para que estos derechos pasen del ideal hacia lo real; esto significa reclamar los derechos humanos en el contexto de los derechos económicos, sociales y culturales. ${ }^{19}$

En la perspectiva de la fundamentación ética del derecho a la soberanía alimentaria, se pueden considerar las contribuciones que se hacen desde la neurobiología. Al respecto, la preocupación por los sentimientos y emociones del otro supera la condición individualista, los cuales deben ser erigidos como normas de comportamiento ético para que se permita la protección y el cumplimiento de derechos básicos para la supervivencia. ${ }^{20}$ Una necesidad básica para la supervivencia, como la de alimentarse, se manifiesta como una exigencia moral del individuo cuando toma conciencia colectiva para

17 Vanegas García, José Hoover, "Ética y derechos humanos en el marco de la Constitución Política de Colombia de 1991", Revista Jurídicas, Colombia, núm 7, enero-junio de 2010, pp. 74-92, disponible en: https://doctrina.vlex.com.co/vid/etica-humanos-mar co-constitucion-politica-232684985 (fecha de acceso: 20 de abril de 2017).

18 Mata Villegas, Nelson, "La educación en la ética de los derechos humanos", Justicia, Colombia, núm. 30, diciembre de 2016, pp. 169-185, disponible en: http://dx.doi. org/10.17081/just.21.30.1357 (fecha de acceso: 23 de abril de 2017), doi: http://dx.doi.org/ 10.17081/just.21.30.1357.

19 Ahlert, Alvori, "Ética y derechos humanos: principios educacionales para una sociedad democrática", Polis, Chile, núm. 16, 2007, pp. 1-13, disponible en: https://jour nals.openedition.org/polis/4663 (fecha de acceso: 12 de diciembre de 2017).

20 Damasio, Antonio, En busca de Spinoza: neurobiología de la emoción y los sentimientos, Barcelona, Crítica, 2005. En esa línea de análisis han trabajado autores como Martínez, Martha y Vasco, Carlos Eduardo, "Sentimientos: encuentro entre la neurobiología y la ética según Antonio Damasio", Revista Colombiana de Bioética, vol. 6, núm 2, diciembre de 2011, planteando la obra de Damasio como un adelanto desde la visión de la condición individual hacia el bienestar social, basados en la ética, elemento de primer orden, apoyándose en los sentimientos y emociones de los otros para buscar un mejor mundo posible para la naturaleza humana. 
construir una ecología de la acción, ${ }^{21}$ pues cuando se realiza una acción individual o colectiva, éstas tienen repercusiones en el entorno e impactan en el mismo; en ese sentido, el autor plantea ir hacia una ética de la sostenibilidad que si bien está basada en la incertidumbre, instaura un principio máximo de responsabilidad. La ética se presenta como una mayúscula creación del ser humano, pues permite entender desde el sentimiento lo que el saber se niega a entender, y es un principio de conmiseración con el otro.

La necesidad de los seres humanos de alimentarse es un principio de autoconservación, ${ }^{22}$ pues no es posible garantizar la vida mientras se sienta hambre, y por ende no se pueden realizar las otras cualidades inherentes al ser humano; esta condición de vulnerabilidad de la corporalidad abre las puertas a que el opresor pueda ejercer control en la medida en que se apodere de los sistemas de producción de los alimentos. De allí la necesidad de una ética de la liberación latinoamericana, ${ }^{23}$ donde el oprimido se hace consciente de la dominación y construye desde el sujeto colectivo, donde se modifica la noción individualista del derecho y se transita hacia un ser ético que considera a la vida como una estética de la existencia dirigiéndose hacia prácticas de vida más amables y conscientes con el entorno.

El sistema jurídico requiere reformas estructurales; empero, es posible trabajar desde un positivismo de combate, pues es a partir de la utilización del mismo derecho oficial como se puede garantizar una materialización al

21 Morin, Edgar, El método 6. Ética, Madrid, Cátedra, 2006. En la misma dirección, Demenchonok, Edward, "Fundamentación de la ética en la filosofía latinoamericana", The Paideia project. Philosophy in Latin America, s.p.i, disponible en: https://www. bu.edu/wcp/Papers/Lati/LatiDeme.htm (fecha de acceso: 7 de noviembre de 2017), indica que la ética debe ocupar un lugar central en la solución de las problemáticas que sólo son posibles desde la autoconciencia y la responsabilidad en las acciones. Esta crisis, en gran parte, es consecuencia de las malas decisiones tomadas desde los gobiernos y con orientaciones internacionales, que optan, como se mencionó anteriormente, por tomar bienes básicos para la supervivencia y otorgárselos a los mercados; no obstante, existe una responsabilidad colectiva, pues se han orientado los modos de vida hacia el egoísmo, el individualismo, la renuncia a las prácticas de vida sustentables, al reconocimiento de la alteridad y a la indiferencia, cuando se trata de la defensa de la vida y del territorio.

22 Dussel, Enrique, Para una ética de la liberación latinoamericana, Buenos Aires, Siglo XXI, 1973.

23 Dussel, Enrique, Ética de la liberación en la edad de la globalización y la exclusión, Madrid, Trotta, 1998. Asimismo, Gudynas, Eduardo, "Buen vivir: germinando alternativas al desarrollo", América Latina en Movimiento (ALAI), núm. 462, febrero de 2011, parte de la idea del buen vivir como el reconocimiento de otra ética para asignar valores, donde la naturaleza está en el centro y no es susceptible de ser mercantilizada. 
acceso a medios dignos de vida, para que se establezca como fin de todos los Estados un derecho a no sufrir de hambre ${ }^{24}$ y éste sea integrado, desde una postura ética, para que no sólo se parta de la protección en un plano meramente antropocéntrico, sino desde un postulado de relación armónica con la naturaleza.

Ejemplos de integración de una relación armónica con la naturaleza se encuentran consignados en la Constitución boliviana desde el suma qamaña que plantea un equilibrio entre el sujeto y el entorno, y desde la Constitución ecuatoriana con el Sumak Kawsay, que se refiere a una vida en plenitud, y al sujeto como parte de la naturaleza. Estos conceptos de buen vivir tienen un origen ancestral y son vistos desde una posición emancipadora que intenta retornar a conceptos planteados antes de la colonia, logrando reconocimiento y acogimiento entre la población de América Latina, pues reconoce la cosmovisión y se instaura desde un discurso legitimador, planteándola como una ética de la sostenibilidad y de la suficiencia para todos.

\section{SEGURIDAD Y SOBERANÍA ALIMENTARIA}

La inquietud ética por la fundamentación del derecho a la soberanía alimentaria no es nueva. El artículo 25 de la Declaración Universal de los Derechos Humanos consagra el derecho a la alimentación. En Colombia, en el mismo sentido se orienta la Constitución Política de Colombia, ${ }^{25}$ que señala explícitamente el derecho a una alimentación adecuada, el cual, sin embargo, para analizar su desarrollo debe ser observado desde dos perspectivas: una es la de la seguridad alimentaria y otra desde la soberanía alimentaria. El sentido que ha prevalecido en Colombia es el de la seguridad alimentaria como garante del derecho a la alimentación, por eso se consagra de la siguiente manera:

Artículo 43. La mujer y el hombre tienen iguales derechos y oportunidades. La mujer no podrá ser sometida a ninguna clase de discriminación. Durante el embarazo y después del parto gozará de especial asistencia y

24 Sen, Amartya, "Propiedad y hambre", Precedente. Revista Jurídica, Colombia, diciembre de 2001, pp. 97-107, disponible en: https://www.icesi.edu.co/revistas/index.php/ precedente/article/view/1384 (fecha de acceso: 16 de mayo de 2017), doi: https://doi.org/ 10.18046/prec.v0.1384.

25 Constitución Política de Colombia, Bogotá, Legis, 1991. 
protección del Estado, y recibirá de éste subsidio alimentario si entonces estuviere desempleada o desamparada.

Artículo 44. Son derechos fundamentales de los niños: la vida, la integridad física, la salud y la seguridad social, la alimentación equilibrada... Gozarán también de los demás derechos consagrados en la Constitución, en las leyes y en los tratados internacionales ratificados por Colombia.

Artículo 46. El Estado, la sociedad y la familia concurrirán para la protección y la asistencia de las personas de la tercera edad y promoverán su integración a la vida activa y comunitaria. El Estado les garantizará los servicios de la seguridad social integral y el subsidio alimentario en caso de indigencia.

Artículo 64. Es deber del Estado promover el acceso progresivo a la propiedad de la tierra de los trabajadores agrarios, en forma individual o asociativa, y a los servicios de educación, salud, vivienda, seguridad social, recreación, crédito, comunicaciones, comercialización de los productos, asistencia técnica y empresarial, con el fin de mejorar el ingreso y calidad de vida de los campesinos.

Artículo 65. La producción de alimentos gozará de la especial protección del Estado. Para tal efecto, se otorgará prioridad al desarrollo integral de las actividades agrícolas, pecuarias, pesqueras, forestales y agroindustriales, así como también a la construcción de obras de infraestructura física y adecuación de tierras. ${ }^{26}$

Organismos multilaterales, como la FAO, también se han ocupado del derecho a la alimentación. Sin embargo, por diversas motivaciones, ha predominado el énfasis en la seguridad alimentaria: "La seguridad alimentaria existe cuando todas las personas tienen, en todo momento, acceso físico, social y económico a alimentos suficientes, inocuos y nutritivos que satisfacen sus necesidades energéticas diarias y preferencias alimentarias para llevar una vida activa y sana". ${ }^{27}$ Enfatizar en la seguridad significa que no importa de dónde provengan los alimentos; mientras éstos puedan ser llevados a los mercados para que la población tenga acceso a ellos, no importa si son importados.

El libre comercio alimentario ha permitido que gran parte de los productos en Colombia sean importados; la Sociedad de Agricultores de Colombia (SAC) calcula que alrededor de 8.8 millones de toneladas de alimentos y

26 Idem.

27 Organización de las Naciones Unidas para la Alimentación y la Agricultura (FAO), Una introducción a los conceptos básicos de la seguridad alimentaria, 1996. 
de materias primas fueron importados para el 2015, mientras que se estima que en Colombia aproximadamente 4.4 millones de personas se encuentran subalimentadas, ${ }^{28}$ y que más de 174 niños menores de 5 años habrían muerto por desnutrición..$^{29}$ Colombia como productor de materias primas se vale del monocultivo para generar más exportaciones y del uso de agrotóxicos para hacer más rentables los cultivos, lo que suscita un desequilibro ambiental.

La preocupación por la alimentación de la humanidad se queda corta bajo el discurso de la seguridad alimentaria. El monocultivo y la confianza en el mercado no han sido suficientes para atender la demanda mundial de alimentos y, por el contrario, ha contribuido a la destrucción de las matrices sociales, culturales y económicas que permiten la producción de alimentos de modo suficiente y en adecuada calidad; las importaciones traen productos a bajo costo y ocasionan un alza en los precios de los productos nacionales, desestimulando al sector agrario, lo que ocasiona, además de una grave problemática ambiental, un impacto social que se evidencia en la migración del campo a la ciudad, generando pobreza y desarraigo rural. La seguridad alimentaria no tiene en cuenta las múltiples culturas que concurren en un territorio, no es de carácter local ni comunitario.

En Colombia, las comunidades rurales del país han logrado posicionar la perspectiva ética que contiene la soberanía alimentaria en el debate constitucional. La Corte Constitucional, al tenor de lo expuesto en la Constitución Política de 1991, estipula el derecho a la alimentación. ${ }^{30}$ La sentencia que

28 Organización de las Naciones Unidas para la Alimentación y la Agricultura (FAO), Mapa del hambre de la FAO, 2015.

29 Instituto Nacional de Salud (INS), Protocolo de vigilancia en salud pública: mortalidad por y asociada a desnutrición en niños menores de 5 años, 2015.

30 En la Sentencia C-644 de 2012 se pronunció en el sentido de que "la seguridad y la soberanía alimentaria, son dimensiones objetiva o institucional y colectiva del derecho fundamental de alimentarse adecuadamente". Este logro, sin embargo, es mínimo frente a toda la arquitectura jurídica que en Colombia beneficia a los organismos transnacionales y que van desde la certificación de las semillas, donde se han dispuesto y ratificado normas como la Decisión 345 de 1993 de la Comunidad Andina que regula el mejoramiento genético de las plantas de 2006; el decreto 4525 de 2005 que facilita la entrada de semillas transgénicas; la Ley 1032 de 2006 que modifica el código penal para penalizar el uso de semillas certificadas o confundibles con éstas; la resolución ICA 187 de 2006 que reglamenta el procesamiento y certificación de la producción agrícola; la resolución ICA 970 de 2010 que continúa la persecución contra los campesinos y el uso de semillas nativas, regulando la propiedad intelectual y las obtenciones vegetales, y la Ley 1518 de 2012 que amplía los alcances de la propiedad intelectual sobre las semillas, por lo tanto trabaja exclusivamente el derecho a la seguridad alimentaria. 
más se acerca a la perspectiva de una fundamentación ética del derecho a la soberanía alimentaria, como enfoque que permite dar cumplimiento al derecho a alimentarse, es la T-348 de 2012, sobre participación de comunidades afectadas por megaproyectos y soberanía alimentaria, que reconoce que al construirse el anillo vial malecón de Crespo, en Bolívar, Colombia, se afectaba el derecho de una comunidad de pescadores a alimentarse y que éstos debían ser consultados para que pudiesen participar de una decisión que les afectaba; con todo y el fallo, el anillo vial fue construido sin estudios previos, lo que ha generado inundaciones y cambios en la cadena trófica de la vida marina, la cual ha sido desplazada del lugar.

La seguridad alimentaria no controla la producción ni fija límites al uso de biotecnologías para el desarrollo de la agricultura; por el contrario, pone al servicio del sector empresarial la producción de alimentos para que éste sea el encargado de diseñar la política agraria que más beneficie la acumulación de capital. ${ }^{31}$ El control trasnacional de los mercados supone una pérdida de la independencia y se vale del dumping en los alimentos para imponerse sobre los mercados locales.

Desde 1996, cuando empezaron a señalarse las directrices en materia de seguridad alimentaria, y a dejar la alimentación de la población mundial en manos del mercado, la Vía Campesina, que es un movimiento internacional que representa alrededor de 200 millones de campesinos en el mundo, ya planteaba el debate entre la acepción de soberanía alimentaria ligada a las lógicas del mercado y la perspectiva ética que permite fundamentar la seguridad alimentaria, ligada a la producción de alimentos desde un estandarte de la lucha por la justicia. La Vía Campesina y otras organizaciones sociales entienden la soberanía alimentaria como:

La soberanía alimentaria es el derecho de cada pueblo a definir sus propias políticas agropecuarias y en materia de alimentación, a proteger y reglamentar la producción agropecuaria nacional y el mercado doméstico a fin de alcanzar metas de desarrollo sustentable, a decidir en qué medida quieren ser autosuficientes, a impedir que sus mercados se vean inundados por productos excedentarios de otros países. ${ }^{32}$

31 Vía Campesina, Declaración del Foro Mundial sobre Agroecología, 2015, disponible en: https://viacampesina.org/es/declaracion-del-foro-internacional-de-agroecologia/ (fecha de acceso: 06 de febrero de 2018).

32 Vía Campesina, Declaración sobre la soberanía alimentaria de los pueblos, Foro Mundial sobre Soberanía Alimentaria, 2002, disponible en: http://movimientos.org/ node/1178? key=1178 (fecha de acceso: 30 de enero de 2018). 


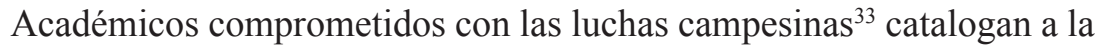
soberanía alimentaria como el mayor reclamo desde las luchas campesinas, y aseguran que un Estado que no enaltece la necesidad de alimentación de su población queda relegado a depender de los caprichos del mercado que regula los precios y puede usarlo a manera de presión sobre los países, tal y como ocurrió con el bloqueo económico impuesto a Cuba desde 1962, de manera que estos autores proponen una reforma agraria verdadera para una agricultura con enfoque agroecológico.

La soberanía alimentaria se fundamenta éticamente, y sus preocupaciones tienen como estandarte la vida, la justicia, la dignidad de los pueblos; por ello, es contraria a las políticas de apertura económica; plantea pensar el territorio desde la multiculturalidad y la autonomía, y que son las comunidades las encargadas de definir de manera concertada sus propias políticas agrícolas, sistemas de producción y de comercialización. Se deslinda de la utilización de semillas certificadas y opta por los cultivos con semillas nativas; se inclina por los mercados locales y la agricultura familiar; por tanto, el monocultivo y la utilización de agrotóxicos no son una opción; la soberanía alimentaria supone la eliminación de la brecha de desigualdad entre el campo y la ciudad; en conclusión, idea una verdadera estética de la vida desde la re-existencia campesina.

\section{RESISTENCIA Y RE-EXISTENCIA DESDE EL TERRITORIO}

Como práctica de la libertad, los pueblos, al tomar sus propias decisiones en materia alimentaria y hacerle frente a la crisis desde una cosmovisión propia que retorna a las prácticas ancestrales, construyen procesos emancipatorios importantes; entre los ejemplos de comunidades que han luchado por la soberanía alimentaria, y que por supuesto no son las únicas experiencias de resistencia y re-existencia campesina en América Latina, se tienen:

En primer lugar, la Vía Campesina como el movimiento internacional campesino que más trabajadores de la tierra aglutina y coordina, tiene un

33 Rosset, Peter y Martínez, María Elena, "Soberanía alimentaria: reclamo mundial del Movimiento Campesino", Ecofronteras, México, vol. XVIII, núm. 51, mayo-agosto de 2014. También se pueden considerar los trabajos de Altieri, Miguel Angel y Nicholls, Clara Inés, Agroecología: unica esperanza para la soberanía alimentaria y la resiliencia, SOCLA, 2012. 
sentido plural, autónomo e independiente. En la búsqueda de una política agraria común, adelanta campañas sobre semillas, reforma agraria y derechos campesinos; además, formula la soberanía alimentaria como uno de sus principios y guía a las asociaciones que la integran hacia el cometido de promoverla en sus territorios.

El Movimiento Sin Tierra de los Trabajadores (MST) ha trabajado fuertemente desde 1984 en contra del latifundismo en Brasil; actualmente es uno de los movimientos campesinos más importantes de América Latina y agrupa poco más de 2 millones de trabajadores de la tierra, los cuales han entendido que la lucha por la soberanía alimentaria es esencial contra el capital corporativo financiero que a través de los organismos multilaterales, llámense Organismo para la Cooperación y el Desarrollo Económicos, Organización Mundial del Comercio, Banco Mundial o Fondo Monetario Internacional, han naturalizado el comercio para que éste sea a favor de las grandes empresas trasnacionales; por eso, uno de los lemas del MST es el de ocupar, resistir y producir; con los últimos gobiernos progresistas en Brasil, han logrado una reforma agraria que les ha permitido trabajar desde sus territorios con un enfoque agroecológico.

El Ejército Zapatista de Liberación Nacional (EZLN) es una organización política y libertaria mexicana que surge a raíz del Tratado de Libre Comercio de América del Norte en 1994, cuando un grupo de campesinos y de comunidades ancestrales se resisten en defensa del territorio; una de sus consignas principales es en pro de la soberanía alimentaria y en contra de la apertura económica que se impulsó en México desde 1982. Sus procesos son autónomos, desde un enfoque agroecológico donde se consume lo que se produce.

En Argentina, se encuentra el Movimiento Campesino de Santiago del Estero (Mocase) creado en 1990; su resistencia se inició a partir de la lucha por la tierra, la reivindicación de los derechos de los trabajadores campesinos y una mejora en las condiciones de vida de los campesinos. $\mathrm{Su}$ enfoque es desde la autogestión, pues consumen solamente lo que producen, al estar integrados por varias familias campesinas y comunidades ancestrales, y tienen gran variedad en la producción de alimentos que intercambian. Una de sus mayores exigencias es en contra del uso abusivo de los recursos naturales.

Entre 1980 y 1987, en Colombia se gestaron las Zonas de Reserva Campesina (ZRC), en respuesta a los reclamos y movilizaciones de los trabajadores campesinos, muy estigmatizadas por su lucha a favor de 
la vindicación de los derechos campesinos en Colombia, en contra del latifundismo, las tierras improductivas y el capital trasnacional. En las ZRC, se trabaja desde las características agrícolas, sociales, económicas y ambientales del territorio; el latifundismo está prohibido y las tierras son solamente para la producción de alimentos. Actualmente se encuentran reglamentadas por la Ley 160 de 1994.

Con la Constitución de 1991 se reconoce a Colombia como un Estado multicultural, lo cual abre un espacio al reconocimiento y a rescatar prácticas de las comunidades ancestrales, que se preocupan por tejer espacios comunitarios autónomos, como los llamados Planes de vida, que procuran una vida armónica con la naturaleza y el respeto máximo a la misma. En dichos planes de vida se trabaja con dos conceptos; uno es el de economía ecológica y el otro es el de etnoecodesarrollo, y con cuatro principios: de autonomía, territorio, unidad y cultura, siempre enalteciendo la memoria colectiva y la solución de conflictos a través del diálogo.

Además de los procesos mencionados, en Colombia se labora por una soberanía alimentaria desde las asociaciones de trabajadores campesinos, las cuales trabajan en cada departamento y producen con enfoques agroecológicos los mercados campesinos como iniciativa de comercialización alternativa de los alimentos, eliminado así a los intermediarios. Se constituyen entonces en una de las experiencias más simbólicas y representativas, cristalizándose en el proyecto de Ley de Desarrollo Rural y Reforma Agraria que pretende el reconocimiento de los derechos de las campesinas y campesinos colombianos.

\section{CONCLUSIONES}

Es posible superar la crisis alimentaria en América Latina si se protege la producción de alimentos desde la perspectiva de una preocupación ética que va más allá de los intereses del mercado y la globalización económica.

La seguridad alimentaria se fundamenta en la lógica del mercado, de allí que desde esta óptica no se puede garantizar el derecho a la alimentación y, por el contrario, se exponga a las comunidades y a los habitantes latinoamericanos a sus azarosos vaivenes.

La soberanía alimentaria, al fundamentarse éticamente, permite la garantía del derecho a la alimentación. De allí que como derecho fundamental es inseparable del concepto de dignidad humana, e implica una perspectiva que 
articula lo universal, lo particular y lo local de la manera como lo ponen en práctica las comunidades campesinas latinoamericanas.

\section{BIBLIOGRAFÍA}

AHLERT, Alvori, "Ética y derechos humanos: principios educacionales para una sociedad democrática", Polis, Chile, núm. 16, 2007, disponible en: https://journals.openedition.org/polis/4663 (fecha de acceso: 12 de diciembre de 2017).

AltiERI, Miguel Angel y Nicholls, Clara Inés, Agroecología: única esperanza para la soberanía alimentaria y la resiliencia, SOCLA, 2012. ÁNGEL, Julialba (comp.), Saber, poder y nuevas formas de lucha en Foucault, Bogotá, Universidad Nacional Abierta y a Distancia, 2016, disponible en: http://hemeroteca.unad.edu.co/index.php/book/article/view/ 1695BIRCH (fecha de acceso: 15 de abril de 2017).

BobBio, Norberto, El tiempo de los derechos, Madrid, Sistema, 1991.

BofF, Leonardo, ¿Economía de revolución?, 2008, disponible en: http:// www.redescristianas.net/2008/08/02/¿economia-de-revolucionleonar do-boff-teologo.

Boff, Leonardo, "El camino más corto hacia el fracaso", Rebelión, España, disponible en: $h t t p: / / w w w . r e b e l i o n . o r g / n o t i c i a . p h p ?$ id $=84428$ (fecha de acceso: 2 de enero de 2018).

CÁRDENAS, Johana y VALLEJO, Luis, "Agricultura y desarrollo rural en Colombia 2011-2013: una aproximación”, Apuntes del Cenes, núm. 62, diciembre de 2016, disponible en: http://www.scielo.org.co/scielo. php script $=$ sci_arttext\&pid $=S 01200532016000200004 \& \operatorname{lng}=e n \& t l$ $n g$ (fecha de acceso: 27 de noviembre de 2017).

Constitución Política de Colombia, Bogotá, Legis, 1991.

DAMASIO, Antonio, En busca de Spinoza: neurobiología de la emoción y los sentimientos, Barcelona, Crítica, 2005.

DÁvalos, Pablo, "Neoliberalismo y Estado social de derecho", América Latina en Movimiento ALAI, Ecuador, junio de 2008, disponible en: http://www.puce.edu.ec/documentos/NeoliberalismoyEstadosocialde derecho.pdf (fecha de acceso: 21 de febrero de 2017).

DEMENCHONOK, Edward, "Fundamentación de la ética en la filosofía latinoamericana", The Paideia project. Philosophy in Latin America, s.p.i, 
disponible en: https://www.bu.edu/wcp/Papers/Lati/LatiDeme.htm (fecha de acceso: 7 de noviembre de 2017 ).

DePARTAMENTO NACIONAL DE ESTADÍSTICA (DANE), Encuesta sobre calidad de vida, 2015.

Dussel, Enrique, Para una ética de la liberación latinoamericana, Buenos Aires, Siglo XXI, 1973, ts. 1 y 2.

DUSSEL, Enrique, Ética de la liberación en la edad de la globalización y la exclusión, Madrid, Trotta, 1998.

GIRALDO, Reinaldo, "La ética en Michel Foucault o de la posibilidad de la resistencia", Tabula Rasa, Colombia, núm. 10, enero-junio de 2009, disponible en: http://www.revistatabularasa.org/numero-10/07giraldo. $p d f$ (fecha de acceso: 13 de marzo de 2018).

GUDYNAS, Eduardo, "Buen vivir: germinando alternativas al desarrollo", América Latina en Movimiento ALAI, Ecuador, núm. 462, febrero de 2011.

HARDT, Michael y NEGRI, Antonio, Imperio, Bogotá, Desde Abajo, 2000.

Informe Nacional de Desarrollo Humano (INDH), Colombia rural: razones para la esperanza, Programa de las Naciones Unidas para el Desarrollo, 2011.

Instituto Geográfico Agustín CodAzzi (IGAC), Atlas de la distribución de la propiedad rural en Colombia, Bogotá, Imprenta Nacional de Colombia, 2012.

Instituto Nacional De SALUd (INS), Protocolo de vigilancia en salud pública: mortalidad por y asociada a desnutrición en niños menores de 5 años, 2015.

KEAN, The neoliberal underpinnings of the bioeconomy: the ideological discourses, 2006.

MARTÍNEZ, Martha y VASCO, Carlos Eduardo, "Sentimientos: encuentro entre la neurobiología y la ética según Antonio Damasio", Revista Colombiana de Bioética, vol. 6, núm 2, diciembre de 2011.

MATA VILlEGAS, Nelson, "La educación en la ética de los derechos humanos", Justicia, Colombia, núm. 30, diciembre de 2016, disponible en: http://dx.doi.org/10.17081/just.21.30.1357 (fecha de acceso: 23 de abril de 2017). doi: http://dx.doi.org/10.17081/just.21.30.1357.

Monedero, Juan Carlos, El gobierno de las palabras. Politica para tiempos de confusión, México, Fondo de Cultura Económica, 2011. 
MorIN, Edgar, El método 6. Ética, Madrid, Cátedra, 2006.

NiETO GóMEZ, Libia Esperanza et al., (In) Certezas del desarrollo: fisuras, relatos y otros senderos, Bogotá, Universidad Nacional Abierta y a Distancia (UNAD), 2014, disponible en: http://hemeroteca.unad.edu.co/in dex.php/book/article/view/1085 (fecha de acceso: 31 de enero de 2018).

OCDE, The Bioeconomy to 2030: Designing a policy agenda, París, OCDE, 2009, https://www.oecd.org/futures/longtermtechnologicalso cietalchallenges/42837897.pdf.

ORGANIZACIÓN DE LAS NACIONES UNIDAS PARA LA ALIMENTACIÓN Y LA AGRICUlTURA (FAO), Una introducción a los conceptos básicos de la seguridad alimentaria, 1996.

ORGANIZACIÓN DE LAS NACIONES UNIDAS PARA LA ALIMENTACIÓN Y LA Agricultura (FAO), Mapa del hambre de la FAO, 2015, disponible en: http://www.fao.org/hunger/es/.

PAVONE, Vincenzo, "Ciencia, neoliberalismo y bioeconomía", Revista Iberoamericana de Ciencia, Tecnología y Sociedad - CTS, Argentina, núm. 20, abril de 2012, disponible en: http://www.redalyc.org/articulo. $o a ? i d=92424169013$ (fecha de acceso: 16 de enero de 2017).

Revista Semana, "Así es la Colombia rural”, Colombia, 2012, disponible en: http://especiales.semana.com/especiales/pilares-tierra/asi-es-la-co lombia-rural.html (fecha de acceso: 5 de marzo de 2018).

RoBInson, James, "La miseria en Colombia", Revista Desarrollo y Sociedad, Colombia, núm. 76, enero-junio de 2016, disponible en: https:// revistas.uniandes.edu.co/doi/pdf/10.13043/dys.76.1 (fecha de acceso: 20 de enero de 2017).

Rosset, Peter y Martínez, María Elena, "Soberanía alimentaria: reclamo mundial del Movimiento Campesino", Ecofronteras, México, vol XVIII, núm. 51, mayo-agosto de 2014.

SÁnCHEZ, Wilson et al., Ontología del presente y construcción de un carácter en Michel Foucault. Discusión a partir del pensamiento de Foucault de los problemas del presente, Santiago de Cali, Universidad Libre, Mariposas Amarillas, 2015.

SEN, Amartya, "Propiedad y hambre", Precedente. Revista Jurídica, Colombia, diciembre de 2001, disponible en: https://www.icesi.edu.co/re vistas/index.php/precedente/article/view/1384 (fecha de acceso: 16 de mayo de 2017), doi: https://doi.org/10.18046/prec.v0.1384. 
VANEGAS GARCÍA, José Hoover, "Ética y derechos humanos en el marco de la Constitución Política de Colombia de 1991", Revista Jurídicas, Colombia, núm 7, enero-junio de 2010, disponible en: https://doc trina.vlex.com.co/vid/etica-humanos-marco-constitucion-politica232684985 (fecha de acceso: 20 de abril de 2017).

Vega CANTOR, Renán, "Crisis civilizatoria", Revista Herramienta, Argentina, núm. 42, octubre de 2009, disponible en: https://www.herra mienta.com.ar/articulo.php? id=1052 (fecha de acceso: 23 de marzo de 2018).

Vía CAmPesina, Declaración del Foro Mundial sobre Agroecología, 2015, disponible en: https://viacampesina.org/es/declaracion-del-forointernacional-de-agroecologia/ (fecha de acceso: 06 de febrero de 2018).

Vía CAMPESINA, Declaración sobre la Soberanía Alimentaria de los Pueblos, Foro Mundial sobre Soberanía Alimentaria, 2002, disponible en: http://movimientos.org/node/1178?key=1178 (fecha de acceso: $30 \mathrm{de}$ enero de 2018).

Fecha de recepción: 26 de septiembre de 2016.

Fecha de aceptación: 22 de febrero de 2019. 